\title{
Hemagglutination of Formalin Preserved Erythrocytes Treated with Tannic Acid and Sensitized with Streptokinase-Streptodoranase (Varidase)
}

\author{
Naohide Kumagar, M.D.* Hiroyoshi Ishil, M.D., \\ and Tachio Kobayashi, M.D.**
}

\begin{abstract}
SUMmary
Hemagglutination of formalin preserved human group $\mathrm{O}$ erythrocytes treated with tannic acid and sensitized with streptokinase-steptodornase (Varidase) were devised by us and investigated in 183 sera of patients suffering from rheumatic fever, acute glomerulonephritis, scarlet fever and chronic tonsillitis etc. High titers were often observed in these poststreptococcal sequelae and titers were commonly low in apparently healthy persons. The titers of the hemagglutination (anti-SK-SD) correlated with that of Todd units of antistreptolysin $\mathrm{O}$, but usually higher than the latter.

It may be useful as one of the serological reactions for the supporting evidence of preceding streptococcal infection in guidance for the diagnosis of rheumatic fever.
\end{abstract}

\section{Additional Indexing Words :}

Anti-streptokinase Anti-streptodornase Rheumatic fever Rheumatic heart disease Jones' criteria Streptococcal infection Streptococcus Glomerulonephritis Scarlet fever Antistreptolysin O

INCE Jones' criteria for guidance in the diagnosis of rheumatic fever was $\checkmark$ revised in $1965,{ }^{1}$ the necessity of the supporting evidence of preceding streptococcal infection has been accentuated and serological reactions to be added to the measurement of antistreptolysin $O$ has been recommended, although they are uncommonly practised because of the technical complexity of the methods.

It is the purpose of this paper to present a new simple method for the measurement of anti-streptokinase-streptodornase (anti-SK-SD) in patientsera and a preliminary report of its clinical applications.

\section{Methods}

Erythrocytes: Human group $\mathrm{O}$ erythrocytes preserved in blood bank were used.

From the Fourth Department of Internal Medicine, (Branch-hospital), the University of Tokyo, Tokyo.

* Assistant Professor of Internal Medicine.

* Professor of Internal Medicine (Director).

Received for publication August 25, 1967. 
Antigens: Varidase (Lederle Co., Ltd. No. 2200-1132-A) was used throughout the experiments. Only for the comparison Lot No. 2200-1141-A and Lot No. 2200-1129-AS were used. *

Formalinization of erythrocytes: Method described by Fauconnier ${ }^{2)}$ and modified by one $\mathrm{e}^{5)}$ of us was used.

Hemagglutination technique: Boyden's tannic acid hemagglutination technique ${ }^{4}$ modified by one ${ }^{31}$ of us was used.

\section{Results}

A. Fundamental studies :

(1) The investigation of influences of concentration of sensitizing antigens upon hemagglutinating titers (Table I and II). The concentration of one vial of Varidase diluted in $10 \mathrm{ml}$. of phosphate buffered saline was most suitable for the sensitization and this concentration was used throughout the experiments. The specificity of the reaction was evidenced by inhibition (Table III).

(2) The reproducibility of the hemagglutinating titers was evidenced as shown in Table IV. The formalin preserved erythrocytes sensitized with antigens were able to be used without changing the efficacy at least one month.

(3) The correlation between the hemagglutinating titers of anti-SKSD and Todd units of anti-streptolysin $O$ was observed (Table V). Titers of anti-SK-SD were usually higher than that of Todd units of anti-streptolysin $O$ in the reciprocal sera.

Table I. Influence of Concentration of Antigen upon Titer of Hemagglutination (I)

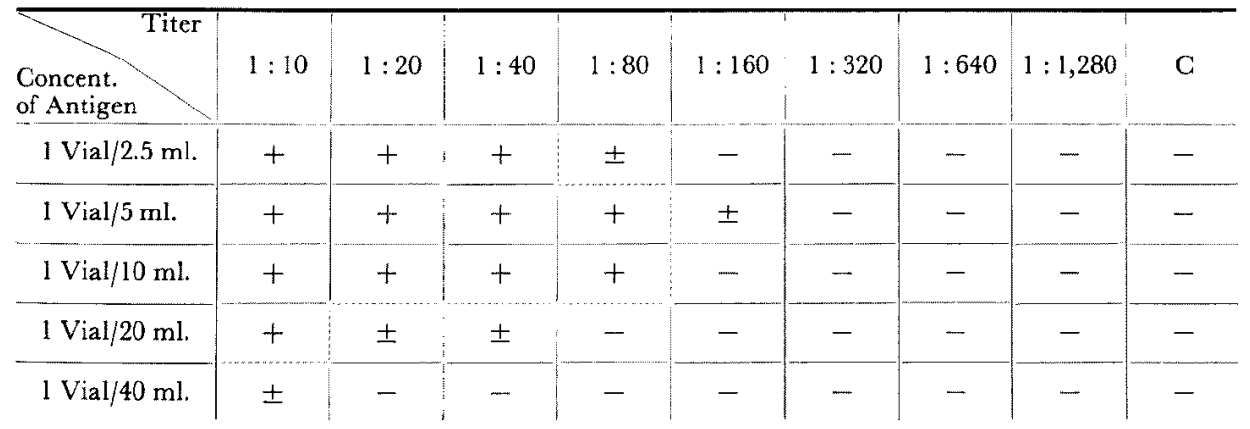

Lot. No. 2200-1132-A..... 1 Vial : SK. 115,686 u. SD. 153,383 u.

*** These vials contain streptokinase and streptodornase as followings according to the data presented by Lederle (Japan), L.td.

$$
\text { Lot 2200-1132-A Lot 2200-1141-A Lot 2200-1129-AS }
$$

SK $115,686 \mathrm{NIH}$ u./vial $102,630 \mathrm{NIH}$ u./vial $122,990 \mathrm{NIH}$ u./vial

SD $153,383 \mathrm{NIH} \mathrm{u./vial} \quad 150,866 \mathrm{NIH}$ u./vial $\quad 87,975 \mathrm{NIH} \mathrm{u} /$ vial 
Table II. Influence of Concentration of Antigen upon Titer of Hemagglutination (II)

\begin{tabular}{|c|c|c|c|c|c|c|c|c|c|c|}
\hline $\begin{array}{l}\text { Tite } \\
\text { of Ancent. }\end{array}$ & $1: 10$ & $1: 20$ & $1: 40$ & $1: 80$ & $1: 160$ & $1: 320$ & $1: 640$ & $1:$ : 1,280 & $\begin{array}{l}1: \\
2,560\end{array}$ & $\mathrm{C}$ \\
\hline 1 Vial $/ 10 \mathrm{ml}$ & + & + & + & + & + & + & + & + & \pm & - \\
\hline $1: 2$ & + & + & + & + & + & + & $++^{\prime}$ & \pm & - & - \\
\hline $1: 4$ & + & + & $t$ & + & + & + & $t^{\prime}$ & \pm & - & - \\
\hline $1: 8$ & + & + & + & $+^{\prime}$ & $t^{\prime}$ & $t^{\prime}$ & \pm & - & - & - \\
\hline $1: 16$ & + & $t^{\prime}$ & +1 & \pm & \pm & - & - & - & - & - \\
\hline $1: 32$ & $+t^{\prime}$ & \pm & \pm & \pm & - & - & - & - & - & - \\
\hline $1: 64$ & \pm & - & - & - & - & - & - & - & - & - \\
\hline $\mathrm{C}$ & - & - & - & - & - & - & - & - & - & - \\
\hline
\end{tabular}

Lot. No. 2200-1132-A.....1 Vial : SK. 115,686 u. SD. 153,383 u.

Table III. Hemagglutination Inhibition Reaction

\begin{tabular}{|c|c|c|c|c|c|c|c|c|}
\hline Antigen & $1: 20$ & $1: 40$ & $1: 80$ & $1: 160$ & $1: 320$ & $1: 640$ & $1: 1,280$ & $\mathrm{C}_{2}$ \\
\hline $1: 40$ & - & - & - & - & - & - & - & - \\
\hline $1: 80$ & - & - & - & - & - & - & - & - \\
\hline $1: 160$ & $+^{\prime}$ & - & - & - & - & - & - & - \\
\hline $1: 320$ & + & $+^{\prime}$ & - & - & - & - & - & - \\
\hline $1: 640$ & + & + & + & - & - & - & - & - \\
\hline $1: 1,280$ & + & + & + & + & $t^{\prime}$ & - & - & - \\
\hline $1: 2,560$ & + & + & + & + & + & - & - & 一 \\
\hline $1: 5,120$ & + & + & + & + & + & - & - & - \\
\hline $1: 10,240$ & + & + & + & + & + & + & - & - \\
\hline $\mathrm{C}_{1}$ & + & + & + & + & + & + & - & - \\
\hline
\end{tabular}

Table IV. Reproducibility of 'Titer of Hemagglutination

\begin{tabular}{|c|c|c|c|c|c|c|c|c|}
\hline Serum $\quad$ Date & $30 / \mathrm{III}$ & $1 / \mathrm{IV}$ & $13 / \mathrm{IV}$ & $15 / 1 \mathrm{~V}$ & $27 / \mathrm{IV}$ & $28 / \mathrm{IV}$ & $3 / \mathrm{V}$ & $7 / \mathrm{V}$ \\
\hline Serum No. 1 & $1: 320$ & $1: 640$ & $1: 320$ & $1: 320$ & $1: 640$ & $1: 320$ & $1: 640$ & $1: 320$ \\
\hline Serum Date & & $4 / I V$ & & & $27 / \mathrm{IV}$ & & & \\
\hline Serum No. 2 & & $1: 160$ & & & $1: 160$ & & & \\
\hline Serum Date & & $11 / \mathrm{IV}$ & & & & & $3 / \mathrm{V}$ & \\
\hline Serum No. 3 & & $1: 320$ & & & & & $1: 320$ & \\
\hline
\end{tabular}


(4) There is no correlation between titers of anti-SK-SD and C.R.P. (Table VI).

B. Clinical Studies:

(1) The distribution of titers in various disease groups was investigated in 183 patient-sera (Table VII). The titers were often extremely high in sera of patients suffering from rheumatic fever, acute glomerulonephritis, scarlet fever and chronic tonsillitis. The titers were usually low in sera of

Table V. Anti-SK.SD and ASLO

\begin{tabular}{|c|c|c|c|c|c|c|c|c|c|}
\hline $\begin{array}{c}\text { ASLO } \\
\text { A-SK.SD }\end{array}$ & $<100$ & 100 & 166 & 250 & 500 & 625 & 833 & 1,250 & $2,500 \leqq$ \\
\hline$\geqq 1: 10,240$ & & & & 0 & & 0 & 000 & 80000 & 0000 \\
\hline $1: 5,120$ & & 0 & 0 & 0 & 0 & & o & & \\
\hline $1: 2,560$ & & 00 & & 80000 & 0 & 0 & $\infty 0$ & 0 & \\
\hline $1: 1,280$ & 0 & 000 & $\infty$ & 80000 & 0 & 80000 & 80000 & O & \\
\hline $1: 640$ & 000 & 80000 & 0 & 80800 & O & 00 & & & 0 \\
\hline $1: 320$ & 80000 & 00000 & 00000 & 80000 & & & & & \\
\hline $1: 160$ & $\begin{array}{l}80 \\
8000 \\
00000\end{array}$ & 0000 & & O & & & & & \\
\hline $1: 80$ & 00000 & 000 & & & & & & & \\
\hline$\geqq 1: 40$ & : & & & o & & & & & \\
\hline
\end{tabular}

Table VI. Anti-SK.SD and C.R.P.

\begin{tabular}{|c|c|c|c|c|c|}
\hline $\mathrm{A}-\mathrm{SK} \cdot \mathrm{SD}$ & $(-)$ & $(+1)$ & $(+2)$ & $(+3)$ & $(+4)$ \\
\hline$\geqq 1: 10,240$ & & & & 0 & \\
\hline $1: 5,120$ & 00 & & & & 0 \\
\hline $1: 2,560$ & 000 & & & & \\
\hline $1: 1,280$ & 80000 & & & & 00000 \\
\hline $1: 640$ & 00000 & & 00 & 0 & \\
\hline $1: 320$ & 88000 & 0 & 0 & 00 & \\
\hline $1: 160$ & 0 & & & 0 & $\mathrm{O}$ \\
\hline $1: 80$ & 0000 & & & & 0 \\
\hline$\leqq 1: 40$ & 00 & & & & \\
\hline
\end{tabular}


patients suffering from other miscellaneous diseases and of apparently healthy persons (donors in blood bank).

(2) The hamagglutination of anti-SK-SD seemed us likely to be useful as one of the supporting evidences of preceding streptococcal infection in guidance for the diagnosis of rheumatic fever (Table VIII).

Table VII. Titers of Anti-SK ·SD Hemagglutination in Various Disease Groups

\begin{tabular}{|c|c|c|c|c|c|c|c|c|c|c|}
\hline Disease $\quad$ Titer & $<40$ & 80 & 160 & 320 & 640 & 1,280 & 2,560 & 5,120 & 10,240 & Total \\
\hline Rheumatic Fever & 1 & & & & 3 & 3 & & 3 & 3 & 13 \\
\hline Rheumatoid Arthritis & & 1 & 2 & 3 & 4 & & 1 & & & 11 \\
\hline Tonsillitis & 3 & 1 & 2 & 6 & 6 & 12 & 4 & & 2 & 36 \\
\hline Rheumatic Heart Disease & 1 & 2 & 3 & 3 & 2 & 6 & 1 & 1 & & 19 \\
\hline Acute Nephritis & & & & & 1 & 2 & & 1 & 3 & 7 \\
\hline Chronic Nephritis & 1 & 1 & & 1 & 1 & 1 & & & & 5 \\
\hline Scarlet Fever & 2 & & & 2 & & 2 & 2 & & & 8 \\
\hline Anaph. Purpura & & & & & 1 & $\mathbb{1}$ & & & 1 & 3 \\
\hline Other Diseases & 7 & 2 & 12 & 8 & 1 & & & & & 30 \\
\hline Gravidity & & & 1 & I & ] & 1 & & & & 4 \\
\hline App. Healthy Persons & 4 & 11 & 12 & 17 & 1 & 2 & & & & 47 \\
\hline
\end{tabular}

Table VIII. Rheumatic Fever

\begin{tabular}{|c|c|c|c|c|c|c|c|c|c|c|c|c|c|}
\hline \multirow{3}{*}{$\begin{array}{r}\text { Symptoms } \\
\text { and } \\
\text { Findings }\end{array}$} & \multicolumn{3}{|c|}{$\begin{array}{c}\text { Major } \\
\text { Manifestations }\end{array}$} & \multicolumn{7}{|c|}{ Minor Manifestations } & \multicolumn{3}{|c|}{$\begin{array}{l}\text { Evidences of Strept. } \\
\text { Infection }\end{array}$} \\
\hline & \multirow[b]{2}{*}{ Carditis } & \multirow{2}{*}{$\begin{array}{l}\frac{5}{5} \\
\frac{\pi}{0} \\
0\end{array}$} & \multirow{2}{*}{$\frac{0}{0}$} & \multirow[b]{2}{*}{ 总 } & \multirow[b]{2}{*}{ a } & \multirow[b]{2}{*}{ Fever } & \multicolumn{3}{|c|}{$\begin{array}{c}\text { Acute Phase } \\
\text { Reaction }\end{array}$} & \multirow[b]{2}{*}{$\begin{array}{l}0 \\
1 \\
=1\end{array}$} & \multirow{2}{*}{$\begin{array}{l}\text { Throat } \\
\text { Culture }\end{array}$} & \multirow{2}{*}{$\frac{0}{1}$} & \multirow{2}{*}{$\begin{array}{l}0 \\
0 \\
\vdots \\
2 \\
2 \\
4\end{array}$} \\
\hline & & & & & & & 范 & 웡 & W.N. & & & & \\
\hline T.O. $10 y \sigma^{*}$ & A.I. & + & - & - & + & $38^{\circ} \mathrm{C}$ & 46 & - & 12,000 & 0.20 & $\begin{array}{c}- \\
\text { Staphyl. }\end{array}$ & 1,250 & $1: 640$ \\
\hline E.N. $13 y$ 우 & $\begin{array}{l}\text { Syst. Mur. } \\
\text { at Apex }\end{array}$ & + & 一 & 一 & + & $39^{\circ} \mathrm{C}$ & 60 & - & 9,600 & w.n.l.* & $\underset{\text { Strept. }}{ \pm}$ & $\begin{array}{r}2,500 \\
<\end{array}$ & $1: 20,480$ \\
\hline M.O. 13y영 & $\begin{array}{l}\text { Syst. Mur. } \\
\text { at Apex. }\end{array}$ & + & - & + & + & $39.5^{\circ} \mathrm{C}$ & 137 & + & 10,900 & w.n.l. & - & 2,500 & $1: 10,240$ \\
\hline T.A. $12 y$ 우 & - & + & + & - & + & $37.5^{\circ} \mathrm{C}$ & 27 & $4+$ & 9,800 & w.n.l. & - & 625 & $1: 1,280$ \\
\hline A.C. $6 y 3$ & $\begin{array}{l}\text { Syst. Mur. } \\
\text { at Apex }\end{array}$ & + & - & - & - & $37.5^{\circ} \mathrm{C}$ & 80 & + & 13,200 & w.n.l. & Cand. & 625 & $1: 1,280$ \\
\hline
\end{tabular}

* whithin normal limits 
Table IX. Rheumatoid Arthritis

\begin{tabular}{|c|c|c|c|c|c|c|c|c|c|c|}
\hline Patient & $\begin{array}{l}\text { mptoms } \\
\text { and } \\
\text { Finding }\end{array}$ & \begin{tabular}{|l|} 
Morning \\
Stiffncss
\end{tabular} & $\begin{array}{l}\text { Joint } \\
\text { Pain }\end{array}$ & $\begin{array}{c}\text { Swelling } \\
\text { of } \\
\text { Joint }\end{array}$ & $\begin{array}{l}\text { Affect. } \\
\text { of } \\
\text { Other } \\
\text { Joint }\end{array}$ & $\begin{array}{l}\text { Symmet- } \\
\text { rical } \\
\text { Affection } \\
\text { of Joints }\end{array}$ & $\begin{array}{c}\text { X-ray } \\
\text { of } \\
\text { Joints }\end{array}$ & $\begin{array}{l}\text { Rh- } \\
\text { factor } \\
\text { (R.A. } \\
\text { Rose) }\end{array}$ & ASL-O & $\begin{array}{c}\mathrm{A}- \\
\mathrm{SK} \cdot \mathrm{SD}\end{array}$ \\
\hline s.o. & $70 \mathrm{y}^{\pi}$ & + & + & + & + & + & Stage 1 & +16 & 500 & $1: 640$ \\
\hline S.W. & $51 y$ 우 & + & + & + & + & + & Stage 3 & +16 & 250 & $1: 640$ \\
\hline T.I. & 76y오 & + & + & + & + & + & Stage 3 & $\begin{array}{r}8 \\
+\quad 4\end{array}$ & $<100$ & $1: 160$ \\
\hline T.K. & $27 \mathrm{y} q$ & + & + & + & + & + & Stage 4 & +16 & & $1: 320$ \\
\hline H.M. & 46y우 & + & + & + & + & + & Stage 4 & +64 & $<100$ & $1: 160$ \\
\hline M.M. & $47 \mathrm{y}$ 우 & + & + & + & + & + & Stage 1 & $+\quad 32$ & 250 & $1: 320$ \\
\hline M.W. & $46 \mathrm{y}$ 우 & + & + & + & + & + & Stage 3 & +32 & 100 & $1: 80$ \\
\hline S.Y. & $52 \mathrm{y}$ 오 & + & + & + & + & + & Stage 3 & + & $<100$ & $1: 320$ \\
\hline
\end{tabular}

Table X. Scarlet Fever

\begin{tabular}{c|c|c|c}
\hline Case No. & Day of Disease & ASLO & Anti-SK $\cdot$ SD \\
\hline 1 & 3 & 250 & $1: 320$ \\
\hline 2 & 3 & 250 & $1: 2,560$ \\
\hline 3 & 5 & 250 & $1: 2,560$ \\
\hline 4 & 5 & 250 & $1: 320$ \\
\hline 5 & 10 & 100 & $<1: 40$ \\
\hline 6 & 12 & $<100$ & $<1: 40$ \\
\hline 7 & 12 & 100 & $1: 640$ \\
\hline 8 & 19 & 100 & $1: 1,280$
\end{tabular}

(3) The titers of anti-SK-SD were not high in sera of patients suffering from definite and classical rheumatoid arthritis (Table IX). This data suggests that rheumatoid arthritis may have less intimate relationship with streptococcal infection than rheumatic fever, as already reported by many investigators and us.

(4) Titers of anti-SK-SD were relatively higher than that of Todd units of antistreptolysin $O$ in sera of 6 in 8 cases of scarlet fever (Table X).

\section{Discussion}

Varidase $^{6)}$ is a commercial product containing streptokinase and strepto- 
dornase prepared from streptococcus group $\mathrm{C}$ and has been used as antigens in classical methods for the measurement of anti-streptokinase in patient sera, for example as Christensen's") method. Recently, the cross-reactivity between streptokinase of streptococcus group $\mathrm{C}$ and that of streptococcus group $\mathrm{A}$ has been discussed by many investigators, ${ }^{81,91,11), 12)}$ and Dilton and Wannamaker ${ }^{11}$ ) have reported that there exists the antigenic similarity between streptokinase of group C and most parts of streptokinase of group A. Therefore, it may be reasonalbe to consider that the titers of the hemagglutination reported in this paper represent the quantities of anti-streptokinase in patient-sera, although anti-streptodornase may influence the titers in sera containing much of antistreptodornase, which may be rare by $\mathrm{K}_{\text {wapirsky }}{ }^{10)}$ and by Wannamaker, ${ }^{13), 14}$ who reported three distinct streptodornase A, B and $\mathrm{C}$ and varidase containing only streptodornase A.

It may be not so difficult to separate streptokinase and streptodornase and to measure anti-streptokinase and anti-streptodornase separately, using this hemaggultination technique. But, for the practical purpose of supporting evidence of preceding streptococcal infection, it may be superior to measure the higher one of the titers of anti-streptokinase and that of anti-streptodornase in patient-sera than to measure these two antibodies separately, because both these extracellular antigens participate simultaneously in practical streptococcal infection and, most of all, it is convenient to use easily attainable commercial product as antigens for the sensitization of erythrocytes in hemagglutination technique.

\section{REFERENGES}

1. Stollerman, G. H., et al.: Circulation 32:664, 1965.

2. Fauconnier, B. : Ann. Inst. Past. 95: 777, 1958.

3. Kumagai, N.: Nisshin-Igaku 47:396, 1960 (in Japanese).

4. Boyden, S. V.: J. Exp. Med. 93 : 106, 1951.

5. Kumagai, N., et al. : Acta Haemat. Japon. 26 : 434, 1963 (in Japanese).

6. Bangham, D. R. and Walton, P. L. : Bull. Wld. Hlth. Org. 33 : 235, 1965.

7. Christensen, L. R.: J. Clin. Invest. 128: 163, 1949.

8. Kaplan, M. H. : Proc. Soc. Exp. Biol. Med. 63: 50, 1946.

9. Weinstein, L. : Proc. Soc. Exp. Biol. Med. 83 : 689, 1953.

10. Kwapinsky, J. B. and Synder, M. L. : Immunology of Rheumatism, Appelton-CenturyCrofts, New York, p. 111, 1962.

11. Dilton, H. C. and Wannamaker, L. W.: J. Exp. Med. 121 : 351, 1965.

12. Houba, V. and Hána, 1.: Immunology 11: 387, 1966.

13. Wannamaker, L. W. : J. Fxp. Med. 107: 797, 1958.

14. Wannamaker, L. W. : Am. J. Med. $27: 567,1959$. 